\title{
Cage Disorder and Gas Encapsulation as Routes to Tailor Properties of Inorganic Clathrates
}

\author{
A. R. Khabibullin ${ }^{1}$, T. D. Huan ${ }^{2}$, G. S. Nolas ${ }^{1}$, and L. M. Woods ${ }^{1, *}$ \\ ${ }^{1}$ Department of Physics, University of South Florida, Tampa, FL 33620, USA \\ ${ }^{2}$ Department of Materials Science \& Engineering and Institute of Materials Science, University of \\ Connecticut, Storrs, CT 06296-3136, USA
}

\begin{abstract}
Inorganic clathrates with the type II crystal structure are of interest as potential materials for high temperature thermoelectric applications. In this study we present ab initio calculations for the electronic and phonon properties of several Sn type II clathrates compositions with partial Ga substitution on the framework, empty cage $\mathrm{Sn}_{136}$, and compounds of $\mathrm{Sn}_{136}$ filled with inert Xe atoms. It is found that cage disorder due to atomic substitution and guest encapsulation affect the fundamental characteristics of these materials in profound ways. We determine that the stability of these materials is enhanced by the presence of guests and lack of direct Ga-Ga bonds in disordered clathrates. Inert Xe atoms provide a unique opportunity to preserve the overall electronic structure of $\mathrm{Sn}_{136}$ and take advantage of the loosely bound guest rattling for enhanced phonon scattering. The calculated energy bands and density of states, as well as phonon band structure and mode Gruneisen parameter, enable further analysis of type II Sn clathrates and reveal interesting structure-property relations.
\end{abstract}

Keywords: clathrates; thermoelectric transport; electronic structure; phonon properties

"Corresponding Author

\section{Introduction}

Clathrate materials have cage-like structures characterized by tetrahedrally coordinated environments with $\mathrm{sp}^{3}$-bonded framework lattices. The family of group IV clathrates and their unique properties have been studied extensively in the past several years, as this interest being motivated by their applications in thermoelectricity, superconductivity, and photovoltaics [1-5]. Inorganic and gas hydrate clathrates are in fact analogous in structure, with classifications of different types available $[1,6]$.

Much of the work to date has focused on the role of the guest atoms inside the clathrate voids. The rattling of such guests (typically alkaline and alkaline-earth species) leads to phonon anharmonic scattering. This is an effective way to reduce the lattice thermal conductivity, a goal that is desirable for thermoelectricity $[1,5]$. The encapsulation of fillers from the first two columns of the periodic table results in ionic bonding with the framework. In many instances, this process reduces the band gap and in some cases it transfers the materials to a metallic state, this being problematic for thermoelectricity. Changing the framework composition is an alternative way of altering their physical properties. This particular direction has only recently begun to be investigated more systematically as the cage atoms can be partially substituted with lower valent atoms, such as Ga, In, and Al $[7,8]$. The mixed cage structural composition is important not only for the electronic properties but also for the phonon dynamics, as shown in type I clathrates $[9,10]$. We note that despite the significant body of research on 
various cage materials in the past several years, more work is needed in order to synthesize clathrates with low thermal conductivity and semiconducting transport for thermoelectric applications.

Exploring clathrates of different compositions shows that the frameworks can be categorized by symmetry of the constituent polyhedra, which can encapsulate different types of guests typically with atoms from group I or II [11]. It is also possible that the guest occupants are methane or noble gas atoms that weakly interact with the framework. The possibility of putting gaseous atoms inside the inorganic clathrate voids can lead to new pathways for property tuning. Due to their weak interaction with the framework, one envisions that Nobel gas inserts may not significantly affect the band structure (thus the semiconducting behavior is preserved), but they can reduce the thermal conductivity at the same time. This would be a much desired scenario for thermoelectricity. Previous investigations have shown that Xe-filled type I clathrates, such as $\mathrm{Xe}_{8} \mathrm{Si}_{46}$, are ultimately unstable [12]. It was also found that the cages of type II clathrates, such as $\mathrm{Si}_{136}$ and $\mathrm{Ge}_{136}$, are generally too small to accommodate inert gas guests [13]. Another possibility to design clathrate properties is to utilize mixed cage materials [14]. Most of the work thus far has focused on type I clathrates [1,9]. However, it would be beneficial to further explore strategies of atomic substitution on frameworks beyond such systems [15] in combination with suitable guest atoms in order to achieve reduced thermal conductivity with a semiconducting transport behavior.

The goal of this research is to advance our fundamental understanding of type II Sn-based clathrates. We note that although type I Sn clathrates have been extensively studied over the past two decades [1618], researchers have only recently begun working on type II Sn materials [19]. To advance the science of these systems and stimulate further experiments, here we investigate several type II Sn clathrates using first principles density functional theory (DFT) simulations. These include the empty cage $\mathrm{Sn}_{136}$, several compounds with partial Ga substitution on the framework with various guest atoms, and several compositions of $\mathrm{Sn}_{136}$ filled with $\mathrm{Xe}$ atoms. Several features found here reveal interesting structureproperty relations, specifically, our simulations clearly show the stabilizing role of the guest atoms, which can also interact strongly with the clathrate framework. It turns out that the location of the Ga atoms on the framework is also a stability factor, which in turn can influence the electronic structure and charge transfer. Of particular interest are the Xe filled $\mathrm{Sn}_{136}$, which show little electronic guest-cage interaction. The phonon properties are also calculated for the various materials. The phonon density of states, phonon band structure, and mode Gruneisen parameter help us give a microscopic picture of the vibrational characteristics of type II Sn clathrates.

\section{Methodology}

Here we perform electronic structure calculations utilizing DFT simulations via the VASP package [20, 21]. This is a state of the art code that relies on the projector-augmented wave method with a planewave basis set and periodic boundary conditions. The exchange-correlation energy is calculated with the Perdew-Burke-Ernzerhof (PBE) function [22]. The unit cells for the considered materials (described in Results and Discussions Section) take advantage of the inherent symmetries of each composition. Ionic relaxation are performed with $367 \mathrm{eV}$ cutoff. The force and total energy difference relaxation criteria are $10^{-4} \mathrm{eV} / \AA$ and $10^{-8} \mathrm{eV}$, respectively. The cell is allowed to change shape and volume during the structural relaxation with $9 \times 9 \times 9 \mathrm{k}$-mesh. The tetrahedron integration method with Blöchl correction is used for the self-consistent calculations on the same k-grid. Also, the VESTA software package is utilized to perform the crystal structure and the electron localization function [23]. 
The phonon properties in terms of vibrational density of states (VDOS) and phonon dispersion spectra for the considered structures are calculated with the PBE functional using the PHONOPY package [24]. For this purpose, finite atomic displacements with an amplitude of $0.01 \AA$ are introduced in the simulated structures. The atomic forces within the supercell are calculated using VASP followed by phonon frequency calculations from the dynamical matrix represented in terms of the force constants. The mode Gruneisen parameter, defined as $\gamma_{i}=-\partial \ln \left(\omega_{i}\right) / \partial \ln (V)$ for each phonon mode with frequency $\omega_{i}(V$ is the volume of the lattice), is also calculated. For this purpose, phonon calculations for the equilibrium volume and two additional volumes that are slightly larger and smaller, are calculated in order to compute the logarithmic derivate in $\gamma_{i}$.

\section{Results and Discussion}

\subsection{Structure and Stability}

Type II clathrates are described by the general chemical formula $A_{8} B_{16} X_{136}(A=C s, R b ; B=N a, K, B a ; X=S i$, Ge or $\mathrm{Sn}$ ) and form in the $F d \overline{3} m$ cubic space group with 136 tetrahedrally coordinated atoms $(\mathrm{X})$ forming the framework of the unit cell with two types of cages: eight hexakaidecahedra $\left[5^{12} 6^{4}\right]$ and sixteen dodecahedra [5 $\left.5^{12}\right]$ [1]. "Guest" atoms can be accommodated inside each cage, where two types of atoms can occupy the different polyhedra. It is also possible to have either $\left[5^{12} 6^{4}\right]$ or $\left[5^{12}\right]$ occupied, or the same type of guest atoms are in both cages. Clathrates with partially substituted cages are feasible as well ( $\mathrm{Y}_{\mathrm{m}} \mathrm{X}_{136-\mathrm{m}}$, where the $\mathrm{Y}$ atoms are also tetrahedrally coordinated in the framework) where charge transfer from the guests to the cage is balanced.

The focus of this investigation are type II Sn-based clathrates. Although $\mathrm{Ba}_{16} \mathrm{Ga}_{32} \mathrm{Sn}_{104}$ with $\mathrm{Ga}$ and $\mathrm{Sn}$ being on the framework have been synthesized almost three decades ago [25], only recently have researchers started investigating these materials. Structural data and transport property measurements of compounds synthesized by various methods have been reported showing intriguing structureproperty relations [18, 26-29]. First principles simulations on a handful of materials have also been performed showing much reduced band gaps and/or transfer to metallic states upon alkaline and earthalkaline atoms cage insertion [30,31]. The phonon dynamics for a few materials has also been reported $[32,33]$.

To obtain broader and more systematic insight into the basic science of these systems we consider $\mathrm{Sn}_{136}$, $\mathrm{Ga}_{40} \mathrm{Sn}_{96}, \mathrm{Cs}_{8} \mathrm{Ba}_{16} \mathrm{Ga}_{40} \mathrm{Sn}_{96}, \mathrm{~K}_{2} \mathrm{Ba}_{16} \mathrm{Ga}_{30} \mathrm{Sn}_{106}, \mathrm{~K}_{8} \mathrm{Ba}_{16} \mathrm{Ga}_{40} \mathrm{Sn}_{96}, \mathrm{Xe}_{8} \mathrm{Sn}_{136}, \mathrm{Xe}_{16} \mathrm{Sn}_{136}$, and $\mathrm{Xe}_{24} \mathrm{Sn}_{136}$. The variety of these compositions gives an excellent opportunity to compare and contrast properties of clathrates with empty cages, partially substituted framework, and cages hosting various guests. Using the inherent symmetries of the $F d \overline{3} m$ space group the conventional unit cell of the empty $\mathrm{X}_{136}$ system can be reduced to a primitive unit cell with 34 atoms, $X_{34}[23,34]$. Similar four-fold reduction can be applied to $A_{8} B_{16} X_{136}$ where the Wyckoff positions for the cage sites are $96 \mathrm{~g}$, 32e and 8a, while the Wyckoff's positions for the guest atoms are $16 \mathrm{c}$ and $8 \mathrm{~b}$. Reducing the number of atoms is especially useful for better computational efficiency. The Ga substitution in the mixed cage systems, however, leads to many possibilities where the $\mathrm{Ga}$ atoms could reside. Considering $\mathrm{Ga}_{40} \mathrm{Sn}_{96}$, for example, there are $\left(\begin{array}{c}34 \\ 10\end{array}\right) \approx 10^{8}$ ways of possible arrangements. For many Ga concentrations the reduction to a primitive cell is not possible, but for a handful of cases $\left(\mathrm{Ga}_{8} \mathrm{Sn}_{128}, \mathrm{Ga}_{32} \mathrm{Sn}_{104}\right.$, and $\left.\mathrm{Ga}_{40} \mathrm{Sn}_{96}\right)$, the construction of a primitive cell can be done. To study these types of arrangements in what follows we take advantage of the primitive unit cell of $\mathrm{Ga}_{40} \mathrm{Sn}_{96}$. We further distinguish between the following possibilities of the $\mathrm{Ga}$ atoms 
arrangements: (i) a substitution by placing the Ga atoms in symmetric 32e and 8a Wyckoff positons; (ii) a random substitution with some direct $\mathrm{Ga}-\mathrm{Ga}$ bonds; (iii) a random substitution with no direct Ga-Ga bond.

Fig. 1 displays some of the studied structures after the $a b$ initio relaxation process. We find that the randomly substituted empty $\mathrm{Sn}_{96}$ framework with $\mathrm{Ga}$ are not stable. A snapshot of the computational process for $\mathrm{Ga}_{40} \mathrm{Sn}_{96}$ at some intermediate step is given in Fig. $1 \mathrm{~b}$ showing distortion of the lattice when compared to the $\mathrm{Sn}_{136}$ system (Fig. 1a). The $\mathrm{Ga}_{40} \mathrm{Sn}_{96}$ eventually becomes severely twisted which leads to breaking of the crystal lattice. Our calculations indicate that the guest atoms in the $\left[5^{12}\right]$ and $\left[5^{12} 6^{4}\right]$ cages remove this distortion and stabilize the structure. Fig. $1 \mathrm{c}$ displays this situation for $\mathrm{Cs}_{8} \mathrm{Ba}_{16} \mathrm{Ga}_{40} \mathrm{Sn}_{96}$ and Fig. 1d shows a larger view of the two types of polyhedra with $\mathrm{Cs}$ and $\mathrm{Ba}$ in each center. We also obtain that the $\mathrm{Xe}_{8} \mathrm{Sn}_{136}, \mathrm{Xe}_{16} \mathrm{Sn}_{136}$, and $\mathrm{Xe}_{24} \mathrm{Sn}_{136}$ have the same structure as that shown one in Fig. 1a with $\mathrm{Xe}$ atoms residing in the center of the respective polyhedra (not shown).

The structural parameters together with the formation energies for several systems are also given in Table 1. The formation energy is defined as $E_{\Delta}=E_{T}-\sum_{i=1}^{n} E_{i}$, where $E_{T}$ is the total energy for the compound and $E_{i}$ is the energy of the constituent atoms ( $n$ - total number of atoms in the cell). According to Table 1 the empty caged Sn clathrate has the smallest lattice constant and nearest neighbor distances, and is the most stable compound. Inserting alkaline and earth-alkaline in cages containing $\mathrm{Ga}$ leads to increasing $a$ and decreasing $E_{\Delta}$. One also finds an increased average Sn-Sn distance when compared to $\mathrm{Sn}_{136}$. The average $\mathrm{Sn}$-Ga distance, on the other hand, is obtained to be about $0.2 \AA$ smaller than the respective to $\mathrm{Sn}$-Sn separation for each material, which is attributed to the smaller $\mathrm{Ga}$ atom. Table 1 further shows that the location of the Ga atom on the framework affects the lattice constant and structural stability. In particular, we obtain that $\mathrm{Cs}_{8} \mathrm{Ba}_{16} \mathrm{Ga}_{40} \mathrm{Sn}_{96}$ has the lowest energy $E_{\Delta}$ when $\mathrm{Ga}$ atoms are randomly distributed with no direct Ga-Ga bond (case (iii)) when compared with cases (i) and (ii). These findings are in agreement with results for type I Ge clathrates, where $\mathrm{Ge}$ atoms with randomly distributed $\mathrm{Ga}$ atoms with no direct $\mathrm{Ga}-\mathrm{Ga}$ bonds also result in a more stable state [35]. Comparing $\mathrm{Cs}_{8} \mathrm{Ba}_{16} \mathrm{Ga}_{40} \mathrm{Sn}_{96}$ and $\mathrm{K}_{8} \mathrm{Ba}_{16} \mathrm{Ga}_{40} \mathrm{Sn}_{96}$ one sees that the lattice constants are very similar, however, the former material is more stable by $0.024 \mathrm{eV} /$ atom. On the other hand the recently studied $\mathrm{K}_{2} \mathrm{Ba}_{14} \mathrm{Ga}_{30} \mathrm{Sn}_{96}$ [25] (for which it is not possible to use symmetric Wyckoff positions in the unit cell) is more stable by $0.118 \mathrm{eV} /$ atom than $\mathrm{K}_{8} \mathrm{Ba}_{16} \mathrm{Ga}_{40} \mathrm{Sn}_{96}$ for which all cages are occupied by the guests.

It is also important to note that Ga distribution on the framework affects the location of the guests. For the case where three is a lack of direct Ga-Ga bonds (iii), Cs and Ba are found in the center of each polyhedron, as shown in Fig. 1d. In the other two cases, however, direct Ga-Ga bonds imply clustering of $\mathrm{Ga}$ atoms on the framework also leading to displacing of the guest atoms from the center of the cages. Specifically for the $\mathrm{Cs}_{8} \mathrm{Ba}_{16} \mathrm{Ga}_{40} \mathrm{Sn}_{96}$ studied here, we find that the displacement of $\mathrm{Cs}$ is $d_{1}=0.29 \AA$ (for case (i)) and $d_{1}=0.63 \AA$ (for case (ii)), while the displacement of $\mathrm{Ba}$ is $d_{2}=0.2 \AA$ (for case (i)) and $d_{2}=0.426 \AA$ (for case (ii)), as shown in Fig. S1 in the Supplementary Information. Similar off-center displacements have been reported in recent experiments concerning type-II Si mixed cage clathrates with vacancies in the framework, where off-center rattling has been considered as an additional factor towards reduction of the thermal conductivity [26]. We suggest that the electrostatic interaction has an important role for these off-center displacements. Following the Zintl-Klemm concept, charge transfer balance between the framework and guest atoms can be achieved and as a result, the Coulomb 
attraction induces a displacement of the guests towards the clustered Ga atoms in the framework (Fig. S1).

The lattice constant-energy relation in systems with Xe atoms encapsulated in the cages is also of interest. Previous authors have shown that type I clathrates cannot accommodate rare-earth gas atoms (with the exception of Ar) due to the relatively small cages formed by Si and Ge clathrates [12, 13, 36]. Nevertheless, this becomes possible for the type II Sn clathrates, as shown here. The lattice constants for all Xe-based clathrates are larger than that for $\mathrm{Sn}_{136}$, and increase as one populates the $\left[5^{12} 6^{4}\right]$ cages $\left(\mathrm{Xe}_{8} \mathrm{Sn}_{136}\right),\left[5^{12}\right]$ cages $\left(\mathrm{Xe}_{16} \mathrm{Sn}_{136}\right)$, or both cages $\left(\mathrm{Xe}_{24} \mathrm{Sn}_{136}\right)$. The same behavior is noted for the nearest neighbor bonds, with $3.053 \AA$ being the largest $\mathrm{Sn}$-Sn distance for $\mathrm{Xe}_{24} \mathrm{Sn}_{136}$. The trend for $E_{\Delta}$ is opposite, decreasing with increasing Xe occupancy. Our results show that the Xe residing in the larger polyhedral $\left(\mathrm{Xe}_{8} \mathrm{Sn}_{136}\right)$ which have the smallest $a$, results in the most stable composition, among the studied Xe filled clathrates.

\subsection{Electronic Structure}

We also investigate the electronic structure of the different materials in terms of energy bands, density of states (DOS) and electron localization function (ELF) calculations. Fig. 2a displays the energy bands and DOS for the empty cage $\mathrm{Sn}_{136}$. This is a semiconductor with a direct gap of $0.327 \mathrm{eV}$ at the L-point. A second direct gap of $0.669 \mathrm{eV}$ is found at the $\Gamma$ point. The obtained gap at the L-point here is larger by $0.135 \mathrm{eV}$ than the one found via DFT-LDA in [29], as is expected due to the well-known fact that LDA methods systematically underestimate energy gaps. Characteristic energy gaps at different high symmetry points in the band structure for $\mathrm{Sn}_{136}$ and several other materials are shown in Table 2 . The $\mathrm{Sn}_{136}$ DOS in the vicinity of $(0,-2.43) \mathrm{eV}$ is primarily composed of $p$ orbitals, followed by a rather large gap in the valence region. The region of $(-3.85,-5.47) \mathrm{eV}$ in DOS is characterized by $s$ and $p$ orbitals due to the $s p^{3}$ - hybridization in the framework (also see Supplemental Material, Fig. S2a). The band structure profile of $\mathrm{Sn}_{136}$ is similar to the one of $\mathrm{Si}_{136}$ and $\mathrm{Ge}_{136}$, which are reported to have energy gaps of 1.24-2.4 eV and 0.7-0.8 eV, respectively [9, 10, 16] (Fig. S2c in the Supplemental Material).

Fig. $2 \mathrm{~b}$ displays the electronic structure of $\mathrm{Cs}_{8} \mathrm{Ba}_{16} \mathrm{Ga}_{40} \mathrm{Sn}_{96}$ with random $\mathrm{Ga}$ atom organization for which symmetry is used to reduce the conventional cell to a primitive unit cell (case (iii)). This DOS shows that placing guest atoms inside the voids has a significant effect on the overall behavior. Specifically, the Ga atoms together with mainly the Ba atoms lead to shifting of the conduction bands downward resulting in a much reduced energy gap at the L-point $(0.171 \mathrm{eV})$. This may be explained by the Zintl concept, which reflects the presence of uncompensated charges in the filled cage clathrate [1,37]. The DOS for $\mathrm{Cs}_{8} \mathrm{Ba}_{16} \mathrm{Ga}_{40} \mathrm{Sn}_{96}$ for cases (i) and (ii) are very similar to the one in Fig. $2 \mathrm{~b}$, however, there is no energy gap found at the Fermi level indicating a metallic state for such structural compositions. Analyzing the obtained DOS in Fig. $2 \mathrm{~b}$ shows that the valence region $(0.0,-2.8) \mathrm{eV}$ is composed of $p$ orbitals from Ga and $\mathrm{Sn}$ atoms. The distinct peak localized in the $(-2.8,-3.2) \mathrm{eV}$ range is due to the contribution from $p$ orbitals of Sn atoms, while $s p^{3}$ hybridization signatures are observed in $(-3.5,-5.5)$ eV (region not shown). The orbital hybridization between $\mathrm{Ba}$ and the nearest Ga atoms occurs in $(-2.82,-3.2) \mathrm{eV}$ and $(0$, 3.43) $\mathrm{eV}$ mainly due to the Ba $s$ and Ga $p$ states. The interaction between $\mathrm{Cs}$ and the nearest $\mathrm{Ga}$ atoms can be traced as hybridization between Cs $s$ and Ga p states in $(-2.31,-1.91) \mathrm{eV}$ and $(0.5,4.02) \mathrm{eV}$. DOS for $\mathrm{K}_{2} \mathrm{Ba}_{14} \mathrm{Ga}_{30} \mathrm{Sn}_{106}$ (ii) (given in Fig. S2d in the Supplementary Information), shows an essentially semimetallic state. The strong hybridization between the $\mathrm{Ga}$ and $\mathrm{Sn}$ state are responsible for the steep peak around $-0.5 \mathrm{eV}$, while the peak around $1.5 \mathrm{eV}$ is an admixture between $\mathrm{Ba}, \mathrm{Ga}$, and $\mathrm{Sn}$. The 
electronic structure $\mathrm{Xe}_{8} \mathrm{Sn}_{136}$ in Fig. 2c shows that the effect of the Xe encapsulation is small on the overall DOS and energy bands composition. It is found that in the depicted energy range, the energy bands and corresponding peaks in DOS are primarily due to the Sn atoms. Nevertheless, the energy gap at the L-point is $0.607 \mathrm{eV}$, which is significantly larger than the L-gap of $\mathrm{Sn}_{136}$. The Xe atom has a significant contribution in the $(-4,-5)$ eV valence region characterized by a localized peak feature, as evident in Fig. S2b.

\subsection{Electron Localization Function}

We further study the nature of chemical bonds and charge transfer characteristics by calculating the electron localization function (ELF) which reflects the probability electron density localization with typical values of $0 \leq \mathrm{ELF} \leq 1$ (ELF $\cong 1$ corresponds to perfect localization, while $\mathrm{ELF} \cong 0.5$ corresponds to an electron gas state) [38]. The ELF results, shown in Fig. 3 for several clathrates, depict the type of bonding in these systems. The red regions in Fig. 3a indicate the covalently shared electrons between the atoms in the $\mathrm{Sn}_{136}$ network. This is very similar to the type of bonding found in $\mathrm{Si}_{136}$. Introducing $\mathrm{Ga}$ into the framework, changes the ELF landscape. The ELF for $\mathrm{Cs}_{8} \mathrm{Ba}_{16} \mathrm{Ga}_{40} \mathrm{Sn}_{96}$, Fig. $3 \mathrm{~b}$, shows that while Sn-Sn have covalent character (similar for Ga-Ga but not shown in Fig. 3) the pronounced red region in vicinity of $\mathrm{Sn}$ indicates polar covalent bonding for Ga-Sn. The interaction between the framework and the Cs and Ba guests is determined to be of ionic character. Ionic bonding between guests from the first two columns of the periodic table and the framework is typical for other clathrates as can be seen in Fig. S3 displaying ELF for $\mathrm{Cs}_{8} \mathrm{Na}_{16} \mathrm{Si}_{136}$. Finally, the ELF results for $\mathrm{Xe}_{24} \mathrm{Sn}_{136}$ are given in Fig. $3 c$ and they are very similar as the ELF for $\mathrm{Xe}_{24} \mathrm{Sn}_{136}$ and $\mathrm{Xe}_{24} \mathrm{Sn}_{136}$ (not shown). Due to the full $3 p^{6}$ shell structure of $\mathrm{Xe}$, no chemical bonding with the framework is expected.

\subsection{Phonon Dynamics}

The calculated vibrational properties of the Sn clathrates include the phonon density of states vDOS, the phonon band structure, and the mode Gruneisen parameter. The calculations rely on obtaining the dynamical matrix $D(q)$, which is then diagonalized within the harmonic approximation to obtain the phonon eigenmodes and eigenvectors. The total and partial vDOS are given in Fig. 4 (bottom panels) for several compounds, while the corresponding phonon band structures are shown in Fig. S5 in the Supplementary Information. The phonon bands allow us to extract the acoustic sound velocities along different k-path directions in the Brillouin zone for the studied materials (given in Table 2). The mode Gruneisen parameter $\gamma_{i}$, calculated within the quasi-harmonic approximation (where frequencies are volume dependent but temperature effects are ignored), is also shown in Fig. 4 (top panels).

Examining the vDOS for $\mathrm{Sn}_{136}$, given in Fig. $4 \mathrm{a}$, shows that acoustic phonons reside in the $(0,1) \mathrm{THz}$ range with sound velocities along the $\Gamma-\mathrm{L}$ and $\Gamma-X$ paths given in Table 2 . The peak centered $\sim 1.5 \mathrm{THz}$ results from several flat optical modes associated with the vibration of the framework. Similar flat modes are responsible for another sharp peak in the $(5,5.5) \mathrm{THz}$ range. The phonon band from acoustic and optical branches along characteristic directions can be also examined in the $\mathrm{Sn}_{136}$ phonon band structure, shown in Fig. S5a in the Supplementary Information. The vDOS for $\mathrm{Cs}_{8} \mathrm{Ba}_{16} \mathrm{Ga}_{40} \mathrm{Sn}_{96}$ in Fig. $4 \mathrm{~b}$ has similarities with the results for the empty cage $\mathrm{Sn}_{136}$ material. The peaks in the $(1,2)$ and $(5,5.5) \mathrm{THz}$ regions are still present, but their sharpness and magnitude are reduced. Fig. $4 \mathrm{~b}$ shows that the vibrations of the $\mathrm{Ba}$ atoms, located in the smaller polyhedra, together with $\mathrm{Sn}$ and Ga contributions from the network determine the vDOS in the $(1,2) \mathrm{THz}$ range, and the peak in the $(5,5.5) \mathrm{THz}$ region is broadened due to the Ga-Sn admixture. Comparing with the results for $\mathrm{Sn}_{136}$, we see that there is an 
additional relatively sharp feature centered at $\sim 0.5 \mathrm{THz}$ in the vDOS, which entirely due to the vibration of the Cs atoms in the larger cages. The corresponding acoustic and flat optical phonon bands are displayed in Fig. S5b. A prominent peak located in $(0.5,0.8) \mathrm{THz}$ region from the vibrations of guests in the larger cages is also present in the vDOS for $\mathrm{Xe}_{8} \mathrm{Sn}_{136}$ and $\mathrm{Xe}_{24} \mathrm{Sn}_{136}$ (Fig. 4c, d), however with much more pronounced sharpness, which is also reflected in the corresponding flat bands in Fig. S5 c,d. The rattling of the Xe atoms in the smaller cages results in two peaks centered at $\sim 1.6$ and $\sim 2 \mathrm{THz}$ with very little admixture from the framework (Fig. 4d). The presence of guest atoms also affects the sound velocities. Table 2 shows that the most dramatic changes are found for $\mathrm{Cs}_{8} \mathrm{Ba}_{16} \mathrm{Ga}_{40} \mathrm{Sn}_{96}$, where along the $\Gamma$-L direction $v_{T R}$ is increased by $197 \mathrm{~m} / \mathrm{s}$, while $v_{L A}$ is decreased by $292 \mathrm{~m} / \mathrm{s}$ when compared with the corresponding values for $\mathrm{Sn}_{136}$. The least dramatic changes are calculated for $\mathrm{Xe}_{8} \mathrm{Sn}_{136}$, where for the $\Gamma-\mathrm{L}$ path $v_{T R}$ is increased by $42 \mathrm{~m} / \mathrm{s}$, while $v_{L A}$ is decreased by $81 \mathrm{~m} / \mathrm{s}$ when compared to $\mathrm{Sn}_{136}$. Very similar values are found for the $\Gamma-X$ direction. The Debye temperatures (averaged for along $\Gamma-L$ and $\Gamma-X$ paths) for the studied materials are also calculated and the results are given in Table 2 . One finds that $\theta_{D}$ is the smallest for the empty cage material and $\theta_{D}$ increases when the lattice constant of the material increases. We further note that the Debye temperature is smaller than the one for clathrates with Ge or Si frameworks as reported in the literature, nevertheless, the values in Table 2 are of similar order as experimental $\theta_{D}$ for type II Sn clathrates [1].

The mode Gruneisen parameter gives further information about the motion of the atoms in the studied materials. It quantifies the stiffness of the bonds as affected by the vibration of the atoms. The top panels in Fig. 4 shows that $\gamma_{i}$ has similar characteristics for all compositions, especially for frequencies larger than $2 \mathrm{THz}$ where $\gamma_{i} \cong$ const. By examining $\omega$ for $\omega<2 \mathrm{THz}$ one finds that there is a localized sharp negative peak at $\omega \sim 0.5 \mathrm{THz}$ corresponding to the $\mathrm{Ba}$ atom vibrations in $\mathrm{Cs}_{8} \mathrm{Ba}_{16} \mathrm{Ga}_{40} \mathrm{Sn}_{96}$ (the larger scale of Fig. $4 \mathrm{~b}$ is given in Fig. S4 in the Supplementary Information). Some negative $\gamma_{i}$ points in a more spread out range are seen for $\mathrm{Sn}_{136}, \mathrm{Xe}_{8} \mathrm{Sn}_{136}$, and $\mathrm{Xe}_{24} \mathrm{Sn}_{136}$ in the $(0.5,2) \mathrm{THz}$ region. The mode Gruneisen parameter for these type II Sn clathrates exhibit similar features those for Si types of clathrates, however the magnitude is larger and the range of negative $\gamma_{i}$ for Si type II systems is much more extended [39]. We note that a negative Gruneisen parameter characterizes negative thermal expansion of a given material, meaning that the material experiences contraction instead of expansion with increasing temperature. It has been suggested that negative thermal expansion may be present at temperatures below room temperature due to the overwhelmingly negative $\gamma_{i}$ for Si clathrates [39] for low $\omega$, however, given that there is mostly positive $\gamma_{i}$, Fig. 4 , we conclude that such a phenomenon is unlikely for Sn clathrates.

The Gruneisen parameter is a quantity that is indicative of the magnitude of the lattice thermal conductivity $\kappa_{L}$. Specifically, in the high temperature regime $\kappa_{L} \sim 1 / \gamma^{2}$, where $\gamma$ is the total Gruneisen parameter. This indicates that in order to achieve low thermal conductivity, the Gruneisen parameter of the material must be large. Within the quasi-harmonic approximation one can show that at lower temperatures $\gamma=\sum C_{V, i} \gamma_{i} / \sum C_{V, i}$, where $C_{V, i}$ is the specific heat at constant volume for each mode. At higher temperatures, however, $C_{V, i}$ approaches the limiting case of the total specific heat $C_{V}$, thus $\gamma=\sum \gamma_{i} / N$, where $N$ is the number of vibrational modes [40]. The so-calculated $\gamma$ for the low and high $\omega$ are shown in Table 2. It is interesting to see that $\gamma$ in the $[2, \infty] \mathrm{THz}$ range, corresponding to higher temperatures, has very similar values for all materials, even though $\gamma$ for $\mathrm{Sn}_{136}$ is the largest. For the $[0,2] \mathrm{THz}$ range, however, the Gruneisen parameters for $\mathrm{Sn}_{136}$ and $\mathrm{Xe}_{8} \mathrm{Sn}_{136}$ are the same, which is larger by $\sim 10 \%$ and $21 \%$ as compared to the calculated values for $\mathrm{Cs}_{8} \mathrm{Ba}_{16} \mathrm{Ga}_{40} \mathrm{Sn}_{96}$ and $\mathrm{Xe}_{24} \mathrm{Sn}_{136}$. 


\section{Conclusions}

Employing ab initio DFT methods we present systematic studies for the basic electronic and phonon properties of type II Sn clathrates. We find that guest atoms are important for the energetic and structural stability of the materials especially for those with frameworks with Ga atom admixtures. Typically alkaline or earth-alkaline atoms result in reduced energy band gap and in some cases a transition to a metallic state can be achieved. The semiconducting type of electronic structure can be preserved by inserting inert gas atoms, such as $\mathrm{Xe}$, whose accommodation becomes feasible due to the large enough Sn cages. The calculated phonon band structure also shows that vibrations from the two different types of polyhedra and the framework have specific signatures. In the vDOS a distinct peak originating from guest atoms vibrating in the larger $\left[5^{12} 6^{4}\right]$ polyhedra is found in the low frequency regime. While this feature has little influence from the framework, the guest vibrations in the smaller $\left[5^{12}\right]$ polyhedra tend to experience significant coupling from the network. The lack of interaction between the Xe atoms and the $\mathrm{Sn}$ framework also results a Gruneisen parameter that is very similar to the one for $\mathrm{Sn}_{136}$. Our investigations also reveal interesting structure-property relations in type II $\mathrm{Sn}$ clathrates, especially in clarifying the stability role of the guest atoms in the different polyhedra and their effects in the electronic and vibrational band structures. Our results will serve as motivation to experimentalists who explore the synthesis of Sn clathrates with inert gases as possible routes to achieve a favorable energy band gap for thermoelectricity while taking advantage of guest atom rattling for reduced thermal conductivity.

\section{Acknowledgements}

We acknowledge financial support from the US National Science Foundation under Grant No. DMR1400957. G.S.N. gratefully acknowledge support from the U.S. Department of Energy, Basic Energy Sciences, Division of Materials Science and Engineering, under Award DE-FG02-04ER46145 for data and results analyses. The use of the University of South Florida Research computing facilities is also acknowledged.

\section{References}

1. G.S. Nolas, The Physics and Chemistry of Inorganic Clathrates (Springer, Berlin, Germany, 2001).

2. F.M. Grosche, H.Q. Yan, W. Carrillo-Cabrera, S. Paschen, C. Langhammer, F. Kromer, G. Sparn, M.

Baenitz, Yu. Grin, F. Steglich, Superconductivity in the filled cage compounds $\mathrm{Ba}_{6} \mathrm{Ge}_{25}$ and $\mathrm{Ba}_{4} \mathrm{Na}_{2} \mathrm{Ge}_{25}$, Phys. Rev. Lett. 87 (2001), 247003.

3. M. Amsler, S. Botti, M.A.L. Marques, T.J. Lenosky, S. Goedecker, Low-density silicon allotropes for photovoltaic applications, Phys. Rev. B 92 (2015), 014101.

4. M. Beekman, New hopes for allotropes, Mat. Today 18 (2015), 304-305.

5. G. S. Nolas, J. W. Sharp, and H. J. Goldsmid, Thermoelectrics: Basic Principles and New Materials Developments (Springer-Verlag, Heidelberg, 2001).

6. W.L. Mao, C.A. Koh, E.D. Sloan, Clathrates hydrates under pressure, Phys. Today 60 (2007), 4247.

7. M. Beekman, G.S. Nolas, Inorganic clathrate-II materials of group 14: systematic routes and physical properties, J. Mater. Chem. 18 (2008), 842-851.

8. S. Bobev, S. Sevov, Clathrates of group 14 with alkali metals: an exploration, J. Solid State Chem. 153 (2000), 92-105. 
9. Y. He, G. Galli, Nanostructured clathrate phonon glasses: beyond the rattling concept, Nano Lett. 14 (2014), 2920-2925.

10. A. Fujiwara, K. Sugimoto, C.-H. Shi, H. Tanaka, J. Tang, Y. Tanabe, J. Hu, S. Heguri, K. Tanigaki, M. Takata, Qualitative relation between structure and thermal conductivity in type-I clathrate $\mathrm{X}_{8} \mathrm{Ga}_{16} \mathrm{Ge}_{30}$ (X=Sr,Ba) based on electrostatic-potential analysis, Phys. Rev. B 85 (2012), 144305.

11. A.J. Karttunen, T.F. Fassler, M. Linnolahti, T.A. Pakkanen, Structural principles of semiconducting group 14 clathrate frameworks, Inorg. Chem. 50 (2011), 1733-1742.

12. X. Blasé, Quasiparticle band structure and screening in silicon and carbon clathrates, Phys. Rev. B 67 (2003), 035211.

13. A. J. Karttunen, T.F. Fassler, Semiconducting clathrates meet gas hydrates: $\mathrm{Xe}_{24}\left[\mathrm{Sn}_{136}\right]$, Chem. A Eur. J. 20 (2014), 6693-6698.

14. Y. Zhang, P.L. Lee, G.S. Nolas, A.P. Wilkinson, Gallium distribution in the clathrate $\mathrm{Sr}_{8} \mathrm{Ga}_{16} \mathrm{Ge}_{30}$ and $\mathrm{Sr}_{4} \mathrm{Eu}_{4} \mathrm{Ga}_{16} \mathrm{Ge}_{30}$ by resonant diffraction, Appl. Phys. Lett. 80 (2002), 2931.

15. J. Martin, S. Erickson, G.S. Nolas, P. Alboni, T.M. Tritt, J. Yang, Structural and transport properties of $\mathrm{Ba}_{8} \mathrm{Ga}_{16} \mathrm{Si}_{x} \mathrm{Ge}_{46-x}$ clathrates, J. Appl. Phys. 99 (2006), 044903.

16. S. Stefanoski, Y. Dong, G.S. Nolas, Structural characterization and low-temperature physical properties of p-type single-crystal $\mathrm{K}_{8} \mathrm{Ga}_{8.5} \mathrm{Sn}_{37.5}$ grown by self-flux method, J. Solid State Chem 204 (2003), 166-169.

17. K. Suekini, M.A. Avila, K. Umeo, H. Fukuoka, S. Yamanaka, T. Nakagawa, T. Takabatake, Simultaneous structure and carrier tunning of dimorphic clathrate $\mathrm{Ba}_{8} \mathrm{Ga}_{16} \mathrm{Sn}_{30}$, Phys. Rev. B 77 (2008), 235119.

18. G.S. Nolas, T.J.R. Weakly, J.L. Cohn, Structural, chemical, and transport properties of a new clathrate compound: $\mathrm{Cs}_{8} \mathrm{Zn}_{4} \mathrm{Sn}_{42}$, Chem. Mater. 11 (1999), 2470-2473.

19. M.C. Schafer, S. Bobev, Tin clathrates with the type II structure, J. Am. Chem. Soc. 135 (2013), 1696-1699.

20. G. Kresse, J. Furthmuller, Efficiency of ab-initio total energy calculations for metals and semiconductors using a plane-wave basis set, Comput. Mater. Sci. 6 (1996), 15-50.

21. G. Kresse, J. Furthmuller, Efficient iterative schemes for ab initio total-energy calculations using a plane-wave basis set, Phys. Rev. B 54 (1996), 11169.

22. P. Perdew, K. Burke, M. Ernzerhof, Atoms, molecules, solids, and surfaces: Applications of the generalized gradient approximation for exchange and correlation, Phys. Rev. Lett. 77 (1996), 3865.

23. K. Momma, F. Izumi, VESTA 3 for three-dimensional visualization of crystal, volumetric and morphology data, J. Appl. Crystallogr. 44 (2011), 1272-1276.

24. A. Togo, I. Tanaka, First Principles Calculations in Materials Science, Scr. Mater. 108 (2015), 1-5.

25. R. Kröner, K. Peters, H.G. von Schnering, R.Z. Nesper, Crystal structure of the clathrate-II, $\mathrm{Ba}_{16} \mathrm{Ga}_{32} \mathrm{Sn}_{104}$, New Cryst. Struct. 213 (1998), 664.

26. S. Mano, T. Onimaru, S. Yamanaka, T. Takabatake, Off-center rattling and thermoelectric properties of type-II clathrate $(\mathrm{K}, \mathrm{Ba})_{24}(\mathrm{Ga}, \mathrm{Sn})_{136}$ single crystals, Phys. Rev. B 84 (2011), 214101.

27. S. Koda, K. Kishimoto, K. Akai, H. Asada, T. Koyanagi, Thermoelectric and transport properties of sintered $\mathrm{n}$-type $\mathrm{K}_{8} \mathrm{Ba}_{16} \mathrm{Ga}_{40} \mathrm{Sn}_{96}$ with type-Il clathrate structure, J. Appl. Phys. 116 (2014), 023710.

28. K. Kishimoto, S. Koda, K. Akai, T. Koyanagi, Thermoelectric properties of sintered type-II clathrates $(\mathrm{K}, \mathrm{Ba})_{24}(\mathrm{Ga}, \mathrm{Sn})_{136}$ with various carrier concentrations, J. Appl. Phys. 118 (2015), 125103. 
29. K. Wei, X. Zeng, T.M. Tritt, A.R. Khabibullin, L.M. Woods, G.S. Nolas, Structure and Transport Properties of Dense Polycrystalline Clathrate-II $(\mathrm{K}, \mathrm{Ba})_{16}(\mathrm{Ga}, \mathrm{Sn})_{136}$ Synthesized by a New Approach Employing SPS, Materials 9 (2016), 732.

30. C.W. Myles, J. Dong, O.F. Sankey, Structural and electronic properties of tin clathrate materials, Phys. Rev. B 64 (2001), 165202.

31. D. Xue, C. Myles, C. Higgins, Effect of guest atom composition on the structural and vibrational properties of the type II clathrate-based materials AxSi136, AxGe136, and AxSn136 ( $A=\mathrm{Na}, \mathrm{K}, \mathrm{Rb}$, Cs; $0 \leq \mathrm{x} \leq 24)$, Materials 9 (2016), 691.

32. C.W. Myles, J. Dong, O.F. Sankey, C.A. Kendziora, G.S. Nolas, Vibrational properties of tin clathrate materials, Phys. Rev. B 65 (2002), 235208.

33. C.W. Myles, J. Dong, O.F. Sankey, Rattling guest atoms in Si, Ge, and Sn-based type-II clathrate Materials, Phys. Stat. Sol. (b) 239 (2003), 26-34.

34. S. Curtarolo, W. Setyawan, G. L.W. Hart, M. Jahnatek, R. V. Chepulskii, R. H. Taylor, S. Wang, J. Xue, K. Yang, O. Levy, M. J. Mehl, H. T. Stokes, D. O. Demchenko, D. Morgan, An automatic framework for high-throughput materials discovery, Comput. Mater. Sci. 58 (2012), 218-226.

35. J. Dong, O. F. Sankey, G. K. Ramachandran, P. F. McMillan, Chemical trends of the rattling phonon modes in alloyed germanium clathrates. J. Appl. Phys. 87 (2000), 7726-7734.

36. D. Connetable, V. Timoshevskii, E. Artacho, X. Blasé, Tailoring band gap and hardness by intercalation: an $a b$ initio study of $\mathrm{I}_{8} @$ Si-46 and related doped clathrates, Phys. Rev. Lett. 87 (2001), 206405.

37. A.V. Shevelkov, K.A. Kovnir, J.V. Zaikina, "Chemistry and Physics of Inverse (Cationic) Clathrates and Tin Anionic Clathrates", 2014, Chapter 5, 125-167 (Springer Series in Materials Science, vol. 199, Netherlands, 2014).

38. G. Frenking, S. Shaik, The Chemical Bond: Fundamental Aspects of Chemical Bonding, (WileyVCH, Weinheim, 2007).

39. V.J. Harkonen, A. J. Karttunen, Ab initio lattice dynamical studies of silicon clathrate frameworks and their negative thermal expandion, Phys. Rev. B 89 (2014), 024305.

40. O. L. Anderson, The Gruneisen ratio for the last 30 years, Geophys. J. Int. 143 (2000), 279-294. 


\begin{tabular}{|c|c|c|c|c|}
\hline Material & $\boldsymbol{a}=\boldsymbol{b}=\boldsymbol{c}[\AA]$ & Sn-Sn $[\AA]$ & Ga-Sn $[\AA]$ & $\boldsymbol{E}_{\Delta}[\mathrm{eV} /$ atom $]$ \\
\hline $\mathrm{Sn}_{136}$ & 17.285 & 2.779 & - & -3.672 \\
\hline $\mathrm{Cs}_{8} \mathrm{Ba}_{16} \mathrm{Ga}_{40} \mathrm{Sn}_{96}$ (i) & 17.389 & - & - & -3.285 \\
\hline $\mathrm{Cs}_{8} \mathrm{Ba}_{16} \mathrm{Ga}_{40} \mathrm{Sn}_{96}$ (ii) & 17.376 & - & - & -3.290 \\
\hline $\mathrm{Cs}_{8} \mathrm{Ba}_{16} \mathrm{Ga}_{40} \mathrm{Sn}_{96}$ (iii) & 17.375 & 2.931 & 2.732 & -3.300 \\
\hline $\mathrm{K}_{2} \mathrm{Ba}_{14} \mathrm{Ga}_{30} \mathrm{Sn}_{106}$ (ii) & 17.485 & 2.922 & 2.749 & -3.379 \\
\hline $\mathrm{K}_{8} \mathrm{Ba}_{16} \mathrm{Ga}_{40} \mathrm{Sn}_{96}$ (i) & 17.387 & 2.929 & 2.727 & -3.261 \\
\hline $\mathrm{Xe}_{8} \mathrm{Sn}_{136}$ & 17.435 & 2.849 & - & -3.449 \\
\hline $\mathrm{Xe}_{16} \mathrm{Sn}_{136}$ & 17.456 & 2.898 & - & -3.211 \\
\hline $\mathrm{Xe}_{24} \mathrm{Sn}_{136}$ & 17.463 & 3.053 & - & -3.047 \\
\hline
\end{tabular}

Table 1 Lattice constants, nearest neighbor bonds, and formation energies for the studied clathrates. The Ga substitution in the cages is denoted as (i) Wyckoff position, (ii) random positions with some direct Ga-Ga bonds, and (iii) random with no direct Ga-Ga bonds. The formation energy per atom in the unit cell is calculated using $E_{\Delta}=E_{T}-\sum_{i=1}^{n} E_{i}$, where $E_{T}$ is the total energy and $E_{i}$ is the energy for constituent atoms). 


\begin{tabular}{|c|c|c|c|c|c|c|c|c|c|c|c|}
\hline \multirow{3}{*}{ Material } & \multirow{2}{*}{\multicolumn{4}{|c|}{ Direct energy gap [eV] }} & \multicolumn{4}{|c|}{ Speed of sound along chosen k-path [m/s] } & \multirow{2}{*}{\multicolumn{2}{|c|}{$\gamma$}} & \multirow{3}{*}{$\boldsymbol{\theta}_{D}[\mathrm{~K}]$} \\
\hline & & & & & & & & & & & \\
\hline & $\mathrm{L}$ & $\Gamma$ & $\mathrm{X}$ & W & $v_{T R}$ & $v_{L A}$ & $v_{T R}$ & $v_{L A}$ & $\begin{array}{c}{[0,2]} \\
\mathrm{THz}\end{array}$ & $\begin{array}{c}{[2, \infty]} \\
\mathrm{THz}\end{array}$ & \\
\hline $\mathrm{Sn}_{136}$ & 0.327 & 0.669 & 1.430 & 1.411 & 1448.75 & 3220.49 & 1450.96 & 3221.87 & 1.42 & 1.54 & 174.2 \\
\hline $\mathrm{Cs}_{8} \mathrm{Ba}_{16} \mathrm{Ga}_{40} \mathrm{Sn}_{96}$ & 0.171 & 0.547 & 1.166 & 0.949 & 1646.27 & 2927.79 & 1647.44 & 2926.85 & 1.28 & 1.49 & 199.3 \\
\hline $\mathrm{Xe}_{8} \mathrm{Sn}_{136}$ & 0.607 & 1.022 & 1.722 & 1.695 & 1491.12 & 3139.17 & 1490.47 & 3138.52 & 1.42 & 1.52 & 180.4 \\
\hline $\mathrm{Xe}_{24} \mathrm{Sn}_{136}$ & 0.430 & 0.541 & 1.124 & 0.968 & 1688.16 & 3127.53 & 1687.55 & 3126.44 & 1.11 & 1.42 & 206.0 \\
\hline
\end{tabular}

Table 2 Direct energy gap at high symmetry points in the Brillouin zone, transverse, $\boldsymbol{v}_{T A}$, and longitudinal, $\boldsymbol{v}_{L A}$, speed of sound along characteristic directions, total mode Gruneisen parameter, $\boldsymbol{\gamma}$, and Debye temperature, $\boldsymbol{\theta}_{\boldsymbol{D}}$ for several type II Sn clathrates are shown. 

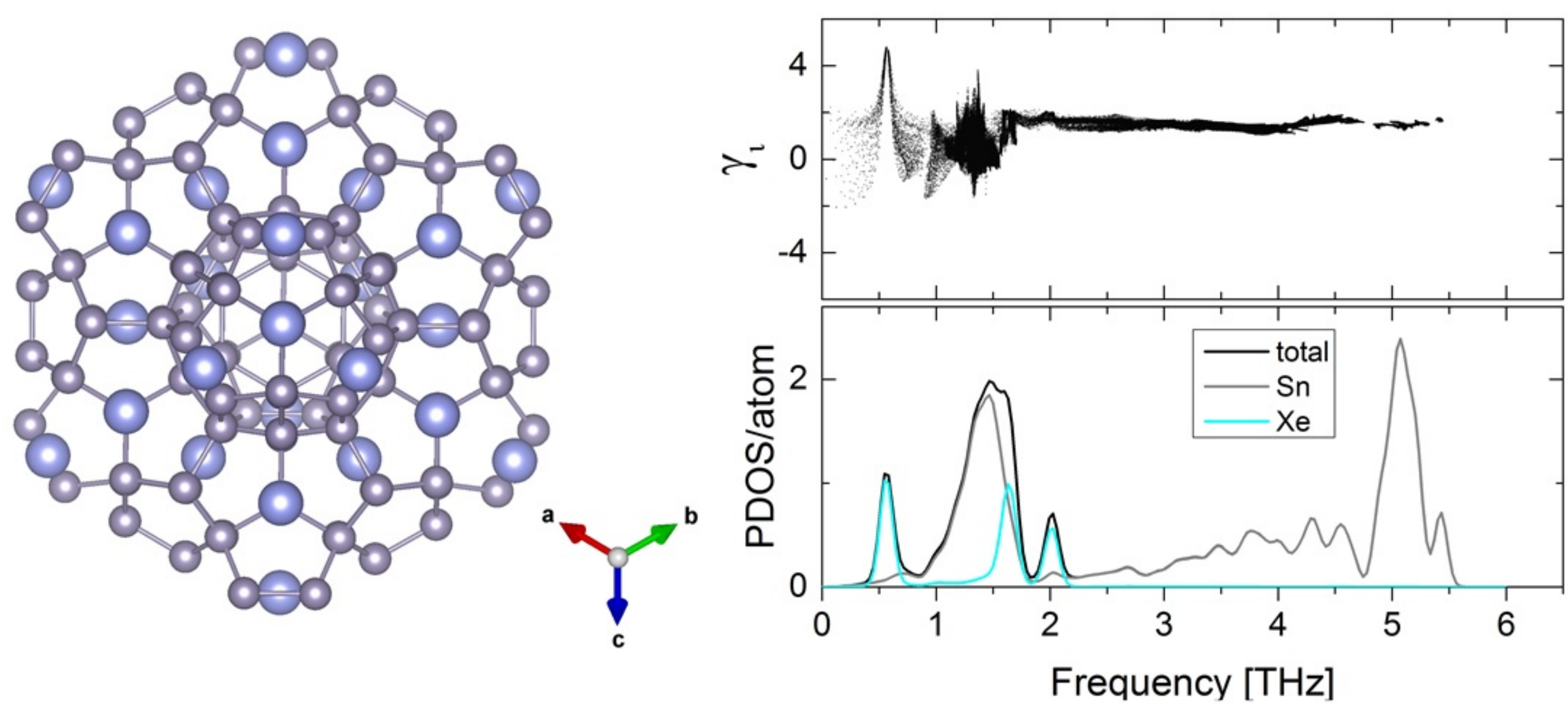

$X e_{24} S n_{136}$ 